\title{
Doing autoethnography of social robots: Ethnographic reflexivity in HRI
}

https://doi.org/10.1515/pjbr-2019-0019

Received September 5, 2018; accepted May 8, 2019

\begin{abstract}
Originating from anthropology, ethnographic reflexivity refers to ethnographers' understanding and articulation of their own intervention to participants' activities as innate study opportunities which affect quality of the ethnographic data. Despite of its methodological discordance with scientific methods which minimize researchers' effects on the data, validity and effectiveness of reflexive ethnography have newly been claimed in technology studies. Inspired by the shift, I suggest potential ways of incorporating ethnographic reflexivity into studies of human-robot social interaction including ethnographic participant observation, collaborative autoethnography and hybrid autoethnography. I presume such approaches would facilitate roboticists' access to human conditions where robots' daily operation occurs. A primary aim here is to fill the field's current methodological gap between needs for better-examining robots' social functioning and a lack of insights from ethnography, prominent socio-technical methods. Supplementary goals are to yield a nuanced understanding of ethnography in HRI and to suggest embracement of reflexive ethnographies for future innovations.
\end{abstract}

Keywords: ethnography in HRI; ethnographic reflexivity in HRI; human-in-the-loop methods in HRI; autoethnography in HRI; the Wizard of $\mathrm{Oz}$ testing; participatory design in HRI; human-robot social interaction; robot sociability; digital anthropology; social robotics

\section{Introduction}

A social robot refers to a robot that is designated to autonomously carry out specific tasks alongside people and to interact socially with them [1]. Based upon the assump-

\footnotetext{
*Corresponding Author: Bohkyung Chun: Department of Anthropology, Oregon State University, USA;

E-mail: chunb@oregonstate.edu
}

tion of robot sociability, the primary goal of HRI research in social robotics is to improve the robot's everyday social functioning. Because roboticists' focus has long been the robot working far away from people [2], the study of human-robot social interaction requires to further explore a variety of techno-social methods that have seldom been appreciated in traditional robotics.

A barrier in such methodological innovations may be involved in the use of relevant methods which conflict with traditional robotics' methodological stance, called positivism in social science theories. The application of ethnographic methods in general is often the case due to their qualitative approach. Especially, ethnographies drawing on the idea of reflexivity entail an extra-difficulty in their incorporation. As I further elaborate in a later section, reflexivity refers to ethnographic researchers' selfawareness and writing of their personal impacts on the quality of the ethnographic data as quintessential study opportunities of ethnographic methods. Reflexive ethnography has widely been appreciated as a productive social method in anthropological technology studies for decades. In addition, despite of its common discordant relationships with scientific methods, its relevance and contribution to understanding advanced human-technology social networks have newly been established over the last decade in the technology field as well, Human-Computer Interaction (HCI) in particular [3, 4].

Inspired by the growing appreciation of reflexivity in ethnographic technology studies, this paper particularly concerns potential implications of ethnographic reflexivity for studies of human-robot social interaction if applied. In HRI, not only is there a lack of ethnography in general, but also there is no ethnography actively embracing reflexivity to date. Thus, a primary aim of this paper is to fill the field's current methodological gap between common needs for better-examining the robot's sociability and a lack of insights from ethnography, widely known as a prominent techno-social method. Supplementary intentions are to develop a nuanced understanding of ethnography as a set of epistemologies rather than simple research techniques and to encourage the field's methodological embracement of ethnographies typically perform- 
ing reflexivity. To achieve these goals, I unfold potential ways and implications of incorporating ethnographic reflexivity into the field by suggesting three paths to applying autoethnography, a typical mode of reflexive ethnography, to studies of human-robot social interaction in a productive manner.

In the following two sections, I first describe ethnography and its reflexivity drawing on anthropological ethnographic theories followed by an outline of both traditional technological ethnography and emerging reflexive ethnography (i.e. autoethnography). Since ethnographic theories are scarce in the current HRI field, I refer to trajectories of technological ethnography found in HCI. Then, I present how performance of reflexivity could generate insights into the development of socially-adaptable robots by suggesting three potential ways of its productive application to studies of human-robot social interaction: participant observation, collaborative autoethnography and hybrid autoethnography. I also discuss the importance of accepting a particular writing style in publishing such autoethnographic studies in the field.

\section{Ethnography and reflexivity}

\subsection{Ethnography}

Ethnography has been used in the technology field for over three decades [5]. Ethnography is, however, still a new method for many roboticists. As someone who has been working in the field with an anthropology background, I begin with a general description of ethnography. I only focus on qualitative ethnography here, because ethnographic studies are predominantly qualitative even though there also are "mixed-method" ethnographies using both the quantitative and the qualitative data [6].

What is ethnography? Ethnography is a primary method of cultural anthropology, which is used to study on social systems and processes shared among specific groups of people [7]. By "social systems," I mean sociocultural and historical circumstances surrounding people which influence the construction of their behaviors, beliefs, self-identities and ideas about others. Meanwhile, by "social processes," I mean ways in which people adjust their behaviors and thoughts in their everyday social lives in relation to social systems. In this sense, a central tenet of ethnography is that human social behaviors and perceptions cannot be understood without the information of their own contexts [8].
In a strategic or technical level, a single ethnographic project always contains two or more data sources to let them validate one another (i.e., triangulation [9]). Hence, ethnography is known as a holistic approach to people and the society. During the period of ethnographic data collection, uniquely called fieldwork, ethnographic researchers directly participate in social groups under study and conduct a series of activities including in-depth interviews, participant observation and visual and textual documentation. In the process of conducting such activities, ethnographic researchers also write and collect their own journals, called fieldnotes, regarding what they learn and think about participants' behaviors and cultures on a daily basis. Simply put, an idea underneath the ethnographic data collection is that the best ways to learn what others do and why is to directly experience their daily lives by interacting with them.

There are some distinctions between traditional and modern ethnography since ethnography dates back hundreds of years. Conventionally, ethnography was assumed to be only used for studying on "far-away" cultures, and fieldwork referred to an ethnographer's physical presence in a culture under study for years. Whereas, in modern ethnography including ethnographic technology studies, ethnographic field sites are not limited to "strangers' worlds" anymore. Ethnographic researchers commonly study on social arenas familiar to themselves such as workplaces, homes, community centers and online social worlds with a shorter duration of fieldwork, e.g., from several weeks to years; it is usual that ethnographers travel to multiple field sites for a single project [10]. In addition, instead of literally moving into a culture under study and staying there for a long period of time, today's ethnographic researchers are more likely to frequently visit participants' mundane lives on a regular basis. Finally, in modern ethnography, it is also common to actively use digital technologies for data collection and rapport creation: participants' activities are photographed and filmed, interviews are conducted via phone and video chat, and social media services are used to develop rapports and to learn participants' updates.

The description of ethnography I made above mostly informs of ethnography as a set of data collection strategies and techniques. Ethnography as a data collection method has been known as being effective and relevant in discovering adaptabilities of computational technologies to everyday human conditions, i.e. how and why certain trends of human behaviors and attitudes towards technological systems occur, vary, persist or change over time [11, 12]. For example, such "in-situ" and "human-inthe-loop" attributes of ethnographic data collection have 
resulted in tech companies' serial employment of anthropologists and increasing "anthro-techno" collaborations in system design.

Meanwhile, there is another dimension of ethnographic methods less known in the technology field. That is, ethnography as a set of epistemologies, i.e. ideas of how we know things [13]. Ethnography does not simply indicate data gathering activities such as observation and indepth interviews but also includes philosophies. Generally speaking, ethnography is often conducted from one of the two contrasting perspectives: positivism and constructivism, i.e. an idea that there is a univocal truth awaiting our discovery VS an idea that the reality is constructed and interpreted uniquely by each person [13]. Most of traditional technological ethnographies rely fairly on the former while ethnographic reflexivity is a concept drawn from the latter. Positivist ethnography, however, also entails reflexivity to some degree in principle. In the following subsection, I further elaborate what reflexivity is, how it is performed and why some may say that even positivist ethnographies are innately reflexive to some degree.

\subsection{Ethnographic reflexivity}

Ethnographic reflexivity refers to ethnographic researchers' awareness and articulation of interpersonal dynamics between researchers and participants and impacts of those subjective dynamics on the research process and outcomes. Since it is one of the core concepts constituting ethnographic methods, there are multiple works theorizing ethnographic reflexivity with distinct accents. This paper primarily borrows Burawoy [14]'s conceptual work of ethnographic reflexivity inspired by Rode [3]'s suggestion of incorporating it within technological ethnography.

First, ethnographic researchers who perform reflexivity admit that their own interventions to participants' actions and reactions create opportunities of data collection as well as impacts on the quality of knowledge produced. In scientific studies, data is collected and tested with a goal of producing unambiguous outcomes, and effects of the individual researcher's actions to the research process are avoided to increase reliability [14]. Whereas, researchers' intervention to and interaction with research participants during the period of data gathering refer to the ethnographic method itself. As long as researchers' interactivity towards studied situations and people generate study opportunities in ethnography, ethnographic researchers' own reflection and interpretation rather increase reliability of ethnographic findings.
In a similar vein, performance of reflexivity implies acknowledgement of ambivalent positional binaries between the researcher and the participant in ethnography drawing back from the position of the authoritative researcher. During fieldwork, ethnographic researchers' and participants' speech acts and experiences are always interrelated and co-created. Namely, ethnography is not simply a method of examining social process, but ethnography itself is also a social process. In many modern ethnographies embracing reflexivity, participants take subjective positions in considering certain study subjects closely related to their lives. Even positivist ethnographies at least depend on the researchers' interactive experiences and personal relationships with their participants (i.e. fieldwork and rapport). Hence, in anthropology and related fields (e.g. cultural studies, gender \& ethnic studies and sociology), there has been a shared understanding that all ethnographic works are intrinsically reflexive and interpretative to some degree although they reveal different levels and styles of reflexivity [4].

Second, practice of reflexivity is closely tied with a question of "how do we tell others about others?" in addition to "how do we know what we know?" while those two questions are ultimately interrelated [15]. Hence, some say "reflexivity is the ethnographer of the text [16]." If ethnographic researchers realized and exploited their positional dynamics with participants in the process of data gathering and analysis, it should be ultimately presented in publications. Reflexive writing aims to tell audiences how the researcher's data gathering impacts the quality of the data itself; it is comparable to reciting the statistical data in quantitative publications. Thus, styles of writing matter to practice of reflexivity in a sense that representation of the reflexive data should be made up of researchers' detailed interpretation and retrospection of their own first hand experiences, interventions and relationships which occurred in the field [3].

Van Maanen [17] classifies ethnographic writing into three styles: the realist tale, the confessional tale, and the impressionist tale. The realist tale foregrounds authenticity of ethnographic representation; it is written in the third person voice as if the researcher was purely an outsiderobserver of the described situation [17]. The realist tale is a style that hardly allows reflexivity since it neither admits interchangeable roles of both the researcher and the participant nor allows interpretative inferences and retrospective episodes. Meanwhile, the confessional tale prioritizes honesty of representation, and it is written in the first person voice in an interpretative manner; it aims to implicate the researcher's own perspective and inference within the account of the data [17]. Lastly, impressionists provide 
a detailed description of impressive episodes experienced by researchers and participants with a limited interpretation; it aims to vividly and dramatically reveal unique attributes of a culture under study by presenting the most striking stories $[15,17]$. The realist tale is the one more likely to be adaptable to the technology field while the confessional and the impressionist tales are rarely found $[3,5]$.

Finally, the concept of reflexivity implies ethnographic researchers who perform reflexivity attempt to find structural patterns in the data, which results in theory building and extension. In other words, the concept legitimizes reflexive insights as valid academic results (i.e. ethnographic publications are neither anecdotal nor personal). It could be said that performance of reflexivity "objectifies" ethnographic insights in an ironical sense.

\section{Reflexivity in technological ethnography}

\subsection{Positivist ethnography in $\mathrm{HCl}$}

It is obvious that reflexivity is an opposite idea to scientific methods. Thus, most of technological ethnography has been positivist ethnography which lacks reflexivity [3]. Such positivist technological ethnography was initiated in the 1980s speaking to the predominant tradition of cognitive science in HCI. Criticizing cognitive science's overfocus on individuals' internal decision-making process in the construction of human reactions towards computer systems, it has widely established the crucial role of contexts in shaping the particulars of human-technology interaction.

There are multiple theoretical frameworks (e.g. situated action [18], activity theory [19] and distributed cognition [19]) advocating distinct understandings of what "context" exactly means as a category of analysis in this ethnographic school [19]. Yet, an overlapped assumption is that ethnography is a method through which researchers objectively measure different contexts of actions conducted by participants who are apart from the researchers themselves. Accordingly, the realist tale is assumed as being the most relevant in conveying the knowledge produced by such ethnography.

For example, "ethnomethodologically-informedethnography [3, 5]" including ethnographies drawing on a concept of "situated action $[18,20]$," maintains that knowledge of the society, e.g. social orders, should be discovered through investigation of people's knowledge of it, i.e. their methods of negotiating it, revealed in their everyday social interactions [21]. Situated action claims that every course of human interaction with the machine is sequentially and minutely created depending upon its situation [18, 20]. Ethnographies drawing on such concepts pay attention to opportunistic and moment-bymoment construction of human reactivity towards the machine, which is a counterpart of cognitive science's separation of the human action from the context $[18,19]$.

When it comes to reflexivity, positivist ethnography also contains reflexivity in a sense that it refuses cognitive science researchers' omnipotence in understanding "knowledge" as transcendental realities; instead, it gives away the authority of knowledge to people's everyday contexts [22]. Positivist ethnography's partial embracement of reflexivity in technology studies corresponds to the statement, "all ethnographies draw on researchers' reflexivity to some degree." Nevertheless, this ethnographic school rarely allows researchers' performance of reflexivity in analysis and writing. For instance, the concept of situated action over-emphasizes improvisatory human actions so that it misses opportunities for building theories regarding more durable trends [19]; it affects a pervasive misunderstanding of ethnography as being anecdotal and unreliable. As another example, a rule of ethnomethodological writing in which participants' description of their own actions can be only accepted as a valid basis forbids researchers' interpretations and inferences in ethnographic writing $[5,22]$.

\subsection{Reflexivity and autoethnography in $\mathrm{HCl}$}

Not to mention HCI researchers' acknowledgement of the nature of ethnographic knowledge production, their emerging attention to ethnographic reflexivity is also involved in the rise of a new paradigm in $\mathrm{HCI}[3,5,23]$. On the one hand, reflexive ethnography has been considered as being useful for those who concern participatory design methods [3]. In spite of its conceptual discordance with traditional scientific methods, reflexive ethnography's focus on the co-creation of both the researcher's and the participant's insights goes well with the trend of participatory design in HCI. On the other hand, a theoretical and analytical shift from "cognitive systems to emotional experiences [4]" in HCI research has also influenced the rise of reflexive ethnography in HCI [23]. When it comes to understanding emotional and experiential dimensions of human-technology interaction, traditional positivist ethnographies are more likely to lose their merits because they mainly burgeoned through a scientific dialogue regarding the construction of human cog- 
nition. Ethnographic studies of emotional experiences require more reflexivity in a sense that reflexivity values ethnographic researchers' emotional and sympathetic approach to potential users' techno-social experiences.

Autoethnography is a typical example of reflexive ethnography which has particularly been wellincorporated into HCI in the context of the field's paradigmatic transformation. Autoethnography refers to an ethnographic approach to research and writing that describe and systematically analyze (i.e., "graphy") ethnographic researchers' personal experiences (i.e. "auto") in order to understand larger cultural structures (i.e. "ethno") [24]. In other words, autoethnography is characterized by the following elements. First, it radically appropriates researchers' practice of reflexivity as a primary data resource of a study. Second, a pivot of autoethnography is an analytical connection between researchers' personal experience and social systems related to it [25]; to increase validity and representativity of the autoethnographic data, auto-ethnographers often conduct in-depth interviews with other social members and reviews of the existing data as well [26]. Lastly, it validates confessional and biographical style of writing in its publications [26].

Besides HCI's emerging focus of participatory and emotional design, another particular reason for the rise of autoethnography in HCI is the methodological efficiency of autoethnography in terms of accessibility to study resources [23]. On a practical level, autoethnography facilitates rapid recruitment of research participants and prolonged observation of participants' private routines. On more inspirational level, there has been a shared understanding that autoethnography provides technology researchers with opportunities for more relevant research design and analysis in studying on other participants' experiences [23]. As autoethnography validates researchers' dual roles as the researcher and the participant, it is no wonder that it encourages researchers' empathetic approach to study subjects. Similarly, it is also claimed that auto-ethnographers are more likely to be sensitive towards ethical issues related to the technology under study as they have a profound understanding of the technology's realworld deployment [27].

According to Rapp [23], such benefits of autoethnography to technology studies have given rise to a cluster of the autoethnographic approach to a variety of study subjects in HCI including impacts of location-based services on a bus drivers' work conditions [28], ways of learning music through listening [29], social interaction of people who do not use smartphones and social media [30], potential everyday functionalities of a wearable device orig- inally designated to increase the awareness of time [31], reliabilities of self-tracking devices [32], the use of a wrist blood pressure among individuals with hypertension [33], early adopters' use of a smartwatch [34] and user cultures of Massively Multi Player Online Role-Playing Games (MMORPGs) [23]. Many of the studies employ autoethnography as means of including researchers as members of participants so that researchers' own interests, motivations and pain points regarding their research subjects are exploited as study resources rather than being remained hidden [35, 36]. Similarly, autoethnography also serves as a theoretical back-up for actual design practice in HCI such as designing a system aiming at supporting users' own reflection and awareness of their own emotional interaction with the system itself [37] and improving a system drawing on the creators' own experience of the system itself [38].

In sum, the growing attention to users' emotional existence in the course of human-technology interaction as well as the crucial role of user-generated insights in system design has engendered needs for alternative methods to the objective and realist approach in HCI. Hence, there has been a reflexive turn of the technological ethnography in HCI. Due to its unique methodological attributes, autoethnography has particularly shown its potential for developing researchers' experiential understanding and sympathetic account of complex techno-social experiences by re-appropriating researchers' personal positionalities as insightful resources rather than obstructive factors to the study. Inspired by such transformation of technological ethnography in HCI, I turn back my gaze upon HRI and suggest three potential paths to incorporating autoethnography into studies of human-robot social interaction in the following section.

\section{Performing ethnographic reflexivity in $\mathrm{HRI}$}

\subsection{From fly-on-the-wall to participant observation}

As a path to validating autoethnography in HRI, I first suggest to broaden the field's current notion of "observation" to include ethnographic participant observation as an option of reliable observation techniques.

Although the number of existing ethnographies in HRI is few, those ethnographies [2, 39] repetitively employ the fly-on-the-wall technique as means of observing participants' reactivities towards robots. The fly-on-the-wall refers to a technique of empirical data gathering originated 
from psychological experimentation, through which the researcher secretly observes behaviors of the researched as if the researcher played a role of a CCTV camera on the wall. The existing ethnographies' exclusive use of fly-onthe-wall technique denotes pervasive methodological hindrance from ethnographic researchers' reflexive practice in HRI research.

A key difference of fly-on-the-wall from ethnographic participant observation is its minimalization of the researcher's involvement in occurring situations. While it is obvious that such observation techniques bring their own values to the study of human social behaviors with a strength of unobtrusiveness, participant observation can also be considered as being valid in HRI research with the concept of reflexivity. It is a basic element of ethnographic fieldwork embracing reflexivity, and even objective ethnographies include participant observation.

If applied to HRI research, participant observation allows the researcher to be physically and socially presenting in the situation of interaction alongside both the participant and the robot. It could offer opportunities for collecting participants' initial thoughts and expressions regarding their interaction with the robot. Through the conduction of participant observation, researchers can make instant conversations with participants and co-experience certain situations of interaction simultaneously with them as well. As long as participant observation is a basic level of reflexive practice in ethnography, acknowledgement of validity and values of participant observation would be a preceding path to incorporating autoethnographic approach within the field.

\subsection{Social robotics teams' collaborative autoethnography}

In traditional sense, autoethnography refers to a singleperson ethnographic researcher's autobiographical investigation of one's own experiences. Whereas, I propose a social robotics team's collective performance of autoethnography regarding their own social interaction with their own robots as a productive way of applying autoethnography to the field; I call it collaborative autoethnography in this paper. Social robotics teams' collaborative autoethnography seems particularly useful in examining pre-released robots' social functioning in everyday laboratory settings throughout development stages. While the previous work mentions the suitability of autoethnography for system design, I suppose that autoethnography, particularly its collaborative variant, would be widely appreciated in HRI as well for the following reasons.
First of all, employment of robots as service providers and co-workers in ordinary human life spheres is still limited despite of a remarkable progress of social robotics technologies over the last decades. At least at this point of innovation, social robotics laboratory and company team members are identified as exceptional groups of people who spend a large portion of their daily lives with robots. In a similar vein, the robot's corporality, the attribute of its physical presence promotes the suitability of autoethnography for HRI even more, compared with other social technologies such as social software and devices. As robots physically hang around in robotics offices and test areas, roboticists have easy access to their own robots particularly when numerous times of testing and quality assurance occur at the early stage. Hence, they are perhaps one of the most eligible population for studies of such novel technologies.

In addition, another potential benefit of collaborative autoethnography is involved in the predominant social and emotional focus of social robotics research in HRI. As mentioned in the preceding section, the autoethnographic approach is characterized by acknowledgement and accommodation of subjectivity, emotionality, and the researcher's influence on the research rather than hiding from these matters [23]. As an intensified way of performing reflexivity, collaborative autoethnography could offer social robotics teams chances of experiential learning regarding robots' sociability. On the one hand, members of collaborative autoethnography would have opportunities for emotionally understanding the robot's social functioning in an embodied manner by reflecting how it's presence in their workplace makes changes in the quality of their everyday social lives. On the other hand, each collaborative auto-ethnographer may also be able to closely observe other co-auto-ethnographers' reactivities towards the robot one another, which preferably leads to iterative discussion of the data during the period of data collection and analysis. In other words, I presume social robotics teams' collaborative autoethnography would work as a participatory design method by identifying individual researcher-participants as sympathetic co-experiencers rather than rational bystanders throughout the process of innovations.

\subsection{From Wizard of $\mathrm{Oz}$ to hybrid autoethnography}

The last suggested path to incorporating autoethnography into HRI in this paper is hybrid autoethnography. Hybrid autoethnography refers to an advanced mode of reframing 
subjective positionalities in HRI research through which traditional positions of the researcher and the study object (i.e. the robot) are mediated and hybridized all together. It aligns the human researcher with the robot as a hybrid entity of observer-actors drawing on both the social robotics' assumption of the robot sociability and the anthropological idea of autoethnography.

Namely, hybrid autoethnography is a conceptual combination of the assumption of robot sociability and autoethnography. Considering that distinct roles of technologies (e.g. tools, media and social actors [40]) differently shape people's relationships and interaction with the technologies, it is inferred that the role of a technology under study constructs the technology's unique relationships with the human researcher, which affects the process of data collection and analysis. The more social robotics technologies evolve, the more positional binaries between the robot and its human counterpart will turn into a fuzzy area. From this perspective, the current positivist approach in HRI seems to contain a methodological inconsistency as it frames the robot as an object in the research process while assuming its subjective social abilities. Here, hybrid autoethnography could offer an alternative framework. Drawing on the autoethnographic idea which metaphorically legitimizes all the research stakeholders' simultaneous performance of dual roles of being the observer and the actor, it methodologically hybridizes the researcher and the robot as mediated observer-actors.

I suppose that hybrid autoethnography would facilitate the advanced application of the Wizard of $\mathrm{Oz}$ technique to the study of robot-prototypes. In the existing ethnography, teleoperation of the robot prototype is conceptualized as wizarding through which teleoperators hide themselves and secretly observe human participants' reactions as a mode of the fly-on-the-wall technique [39]. The Wizard of $\mathrm{Oz}$ approach identifies teleoperating researchers as omnipotent third-person experimenters rather than integral actors. While the technique has many advantages such as facilitating low-cost evaluation of the technology at an early-development stage and providing teleoperating researchers with insights into technologies' real-world adaptability, it also has disadvantages such as difficulties in faking technologies, playing consistent wizarding skills and pursuing transparency of the data gathering process.

Hybrid autoethnography would transform such shortcomings into methodological strengths in teleoperation of the robot prototype. Unlike the Wizard of Oz's prohibition of reflexivity, hybrid autoethnography allows a researcher's autoethnographic performance of reflexivity legitimizing the researcher (i.e. teleoperator) "to become a robot (i.e. a social actor in the field)" rather than to control the robot. For example, teleoperators' failure of wizarding robot-like behaviors or their own improvised wizarding is not a risk but an opportunity in hybrid ethnography because such incidents become a part of experiential learning process of hybrid auto-ethnographers who play a role of the social actor (i.e. the robot) in the situation of interaction. Even if teleoperation guidelines are given, individual operators' personal effects and opportunistic teleoperating behaviors still serve as the valid data supposed to be reflected and critically analyzed by the hybrid autoethnographer. Plus, the social presence of the researcher in the situation of interaction increases the research transparency. In this sense, I suppose that hybrid autoethnography would be a method which keeps reflexive abilities of the human researcher as well as social abilities of the robot in the loop by assigning them the hybrid positionality of observer-actors in the course of data collection and analysis.

\section{Conclusion}

While the positivist ethnography has long been considered as being the most appropriate for technology studies, there has also been a growing attention to the value of reflexive ethnography in the technology field. Inspired by the shift, this paper is a methodological exploration of incorporating autoethnography, a typical reflexive ethnography, within studies of human-robot social interaction. Suggesting three potential paths to the productive application of autoethnography including participant observation, collaborative autoethnography and hybrid autoethnography, I discuss that autoethnography legitimates researchers' physical and social participation in the situation of interaction so that it lowers their barrier-to-entries to everyday settings of the robot's operation and people's latent needs for the robot.

Considering the current restriction towards ethnographic reflexivity in HRI research, primary contributions of this paper could be to suggest customized paths to the incorporation of reflexive ethnography as well as nuanced understanding of it as a relevant techno-social method in the field. On the other hand, one thing that is not thoroughly suggested and articulated in this paper is the importance of incorporating autoethnographic writing styles corresponding to such paths. The incorporation of reflexive writing into the field would be more challenging than the accommodation of reflexive data gathering and analysis considering the predominant scientific standard 
of publications in HRI and robotics. Nevertheless, another final and primary suggestion of this paper is the field's development and embracement of its own reflexive textualization styles well-representing individual autoethnographers' retrospective, interpretative and inferential voices as well as autoethnographic research procedures and strategies used.

\section{References}

[1] T. Fong, I. Nourbakhsh, K. Dautenhahn, A survey of socially interactive robots, Robotics and Autonomous Systems, 2003, 42(3-4), 143-166

[2] A. Sauppé, B. Mutlu, The social impact of a robot co-worker in industrial settings, In: Proceedings of ACM International Conference on Human Factors in Computing Systems, 2015

[3] J. Rode, Reflexivity in digital anthropology, In: Proceedings of the ACM SIGCHI Conference on Human Factors in Computing Systems, 2011

[4] S. Bødker, When second wave $\mathrm{HCl}$ meets third wave challenges, In: Proceedings of the 4th Nordic ACM Conference on HumanComputer Interaction: Changing roles, 2006

[5] A. Crabtree, T. Rodden, P. Tolmie, G. Button, Ethnography considered harmful, In: Proceedings of the SIGCHI Conference on Human Factors in Computing Systems, 2009, 879-888

[6] M. Hammersley, P. Atkinson, Ethnography: Principles in Practice, Routledge, 2007

[7] P. Atkinson, Ethnography, In: P. Atkinson (Ed.), Routledge Handbook of Qualitative Research in Sport and Exercise, Routledge, 2016, 71-83

[8] J. Boyle, Styles of ethnography, Critical Issues in Qualitative Research Methods, 1994, 2, 159-185

[9] P. Rothbauer, Triangulation, In: L. Given (Ed.), The SAGE Encyclopedia of Qualitative Research Methods, Sage Publications, 2008, 892-894

[10] U. Hannerz, Being there... and there... and there! Reflections on multi-site ethnography, Ethnography, 2003, 4(2), 201-216

[11] B. Nardi, The use of ethnographic methods in design and evaluation, In: B. Nardi (Ed.), Handbook of Human-Computer Interaction, 1997, 361-366

[12] J. Hughes, Moving out from the control room: ethnography in system design, In: Proceedings of the ACM Conference on Computer Supported Cooperative Work, 1994

[13] R. Bernard, C. Gravlee, Handbook of methods in cultural anthropology, Rowman \& Littlefield, 2014

[14] M. Burawoy, The extended case method, Sociological Theory, 1998, 16(1), 4-33

[15] A. N. Markham, Ethnography in the digital Internet era: From fields to flow, descriptions to interventions, In: N. Denzin, Y. Lincoln (Eds.), The Sage Handbook of Qualitative Research, Sage Publications, 2017

[16] S. Woolgar, Reflexivity is the ethnographer of the text, In: S. Woolgar (Ed.), Knowledge and Reflexivity, New Frontiers in the Sociology of Knowledge, Sage Publications, 1988, 14-35

[17] J. Van Maanen, Tales of the Field: On Writing Ethnography, University of Chicago Press, 1988
[18] L. Suchman, Plans and Situated Actions: The Problem of HumanMachine Communication, Cambridge University Press, 1987

[19] B. Nardi, Studying context: A comparison of activity theory, situated action models, and distributed cognition, Context and Consciousness: Activity Theory and Human-Computer Interaction, 1996, 69-102

[20] L. Suchman, Lucy, Reconstructing technologies as social practice, American Behavioral Scientist, 1999, 43(3), 392-408

[21] H. Garfinkel, Studies in Ethnomethodology, Englewood Cliffs, 1984

[22] P. A. Carbó, Reflexive practice in the ethnographic text: Relations and meanings of the use of heroin and other drugs in an urban community, Forum: Qualitative Social Research, 2009, 10(2), Art. 23

[23] A. Rapp, Autoethnography in Human-Computer Interaction: Theory and Practice, In: New Directions in Third Wave HumanComputer Interaction: Volume 2 - Methodologies, Springer, 2018, 25-42

[24] P. Dourish, Implications for design, In: Proceedings of the ACM SIGCHI Conference on Human Factors in Computing Systems, 2006, 541-550

[25] C. Ellis, Carolyn, The Ethnographic I: A Methodological Novel about Autoethnography, Alta Mira Press, 2004

[26] C. Ellis, T. Adams, A. Bochner, Autoethnography: An overview, Historical Social Research, 2011, 273-290

[27] L. Malinverni, N. Pares N, An autoethnographic approach to guide situated ethical decisions in participatory design with teenagers, Interact Computer, 2017, 29(3), 403-415

[28] G. Pritcherd, J. Vines, P. Briggs, L. Thomas, P. Olivier, Digitally driven: how location based services impact on the work practices of London bus drivers, In: Proceedings of the ACM SIGCHI Conference on Human Factors in Computing Systems, 2014, 3617-3626

[29] X. Xiao, H. Ishii, Inspect, embody, invent: a design framework for music learning and beyond, In: Proceedings of the ACM CHI Conference on Human Factors in Computing Systems, 2016, 5397-5408

[30] L. Diaz, C. Morales, L. Gaytan-Lugo, V. Cid, L. Morales, S. Enriquez, Living without a smartphone: using autoethnography to get closer to basic phone users, Paper presented at CHI 2017 symposium on $\mathrm{HCl}$ across Borders Denver, 2017, 6-7

[31] D. Harrison, M. Cecchinato, Give me five minutes!" Feeling time slip by, In: Adjunct Proceedings of the 2015 ACM International Joint Conference on Pervasive and Ubiquitous Computing and Proceedings of the 2015 ACM International Symposium on Wearable Computers, 2015, 45-48

[32] A. Marcengo, A. Rapp, F. Cena, M. Geymonat, The falsified self: complexities in personal data collection, In: Proceedings of the ACM HCl International Conference, 2016, 351-358

[33] A. O'Kane, Y. Rogers, A. Blandford, Gaining empathy for nonroutine mobile device use through autoethnography, In: Proceedings of the ACM SIGCHI Conference on Human Factors in Computing Systems, 2014, 987-990

[34] M. Cecchinato, A. Cox, J. Bird, Always on(line)? User experience of smartwatches and their role within multi-device ecologies, In: Proceedings of the 2017 ACM CHI Conference on Human Factors in Computing Systems, 2017, 3557-3568

[35] S. Ljungblad, Passive photography from a creative perspective: "if I would just shoot the same thing for seven days, it's like... what's the point?," In: Proceedings of the ACM SIGCHI Confer- 
ence on Human Factors in Computing Systems, 2009, 829-838

[36] K. Williams, An anxious alliance, Aarhus Series on Human Centered Computing, 2015, 1(1), 11

[37] K. Boehner, P. Sengers, S. Warner, Interfaces with the ineffable: meeting aesthetic experience on its own terms, ACM Transaction of Computer Human Interaction, 2008, 15(3),1-29

[38] C. Neustaedter, P. Sengers, Autobiographical design: what you can learn from designing for yourself, Interactions, 2012, 19(6), 28-33
[39] A. Sabelli, A. Maria, T. Kanda, N. Hagita, A conversational robot in an elderly care center: an ethnographic study, In: Proceedings of the ACM Conference on Human-Robot Interaction, 2011

[40] B. Fogg, Persuasive computers: Perspectives and research directions, In: Proceedings of ACM CHI Conference on Human Factors in Computing Systems, 1998, 225-232 